\title{
Références bibliographiques du dossier « Conflits de vérités à l'école »
}

Hélène Beaucher

\section{OpenEdition \\ 12 Journals}

Édition électronique

URL : https://journals.openedition.org/ries/6160

DOI : $10.4000 /$ ries. 6160

ISSN : 2261-4265

Éditeur

France Education international

\section{Édition imprimée}

Date de publication : 30 avril 2018

Pagination : 157-168

ISBN : 978-2-85420-618-0

ISSN : 1254-4590

Référence électronique

Hélène Beaucher, «Références bibliographiques du dossier "Conflits de vérités à l'école » ", Revue internationale d'éducation de Sèvres [En ligne], 77 | avril 2018, mis en ligne le 30 avril 2018, consulté le 25 juin 2021. URL : http://journals.openedition.org/ries/6160 ; DOI : https://doi.org/10.4000/ries.6160 


\title{
Références bibliographiques du dossier « Conflits de vérités à l'école »
}

\section{Hélène Beaucher}

CIEP

\begin{abstract}
Quelle(s) vérités enseigne l'école? Sont-elles contestées? Comment sont-elles travaillées dans le système scolaire ? Y a-t-il conflit entre des régimes et de vérité ? Quelles sont les pratiques des enseignants face à la controverse?
\end{abstract}

Enjeux épistémologiques, enjeux didactiques, enjeux disciplinaires, question des identités, des croyances, du pouvoir, conflits de valeurs, etc. : les problématiques et enjeux soulevés par le sujet, miroir de la complexité des sociétés contemporaines, sont nombreux et il est impossible de les traiter de manière approfondie dans le cadre d'une bibliographie.

Les premières références ont pour objet : vérité et rationalité en philosophie de la connaissance, vérité et opinion, vérité et éducation, diversité culturelle et religieuse à l'école. La partie suivante traite des polémiques et propose des exemples de tensions entre régimes de vérité scientifique et religieux, et de questions sensibles en histoire. Un troisième ensemble porte sur l'enseignement des questions socialement vives et sur les pratiques des enseignants face à la controverse; aborder ce qui fait débat permet de renforcer les compétences civiques. La dernière partie s'intéresse à l'éducatio n à la citoyenneté, qui contribue à l'apaisement des conflits et favorise le vivre-ensemble et l'acquisition de valeurs communes.

Cette bibliographie concerne en majorité des travaux produits ces dix dernières années.

\section{VÉRITÉ ET RATIONALITÉ EN PHILOSOPHIE DE LA CONNAISSANCE}

\section{BARREAU Hervé, L'épistémologie, PUF/Paris, 2013, 128 p.}

L'épistémologie est l'étude de la science, ou plutôt des sciences. Elle implique que la connaissance scientifique, de même que la connaissance commune sur laquelle elle s'appuie, se situent toutes deux dans l'Histoire. Entre cette base, et son environnement social, culturel et éthique, se situe l'éventail entier de la connaissance scientifique. Cet ouvrage analyse l'ensemble des problèmes qu'elle soulève, de la logique aux sciences de l'homme et de la société, en passant par les sciences physiques et les sciences du vivant.

DEBRU Claude, KAHANE Jean-Pierre, SANCHEZ-PALINCIALA (dir.), La rationalité scientifique aujourd'hui : rapport de l'Académie des sciences, Edp Sciences/ Les Ulis, 2016, 98 p.

Qu'en est-il de la rationalité scientifique aujourd'hui, alors que les questions de société ont un rôle de plus en plus déterminant dans les orientations des sciences et dans la conception de ce qu'elles sont par rapport à d'autres activités sociales? Des spécialistes de disciplines variées réfléchissent sur leurs démarches propres et sur les voies de leur créativité. 
LADRIERE Jean, Les enjeux de la rationalité : le défi de la science et de la technologie aux cultures, Liber/Québec, 2001, 290 p.

Science et technologie affectent les cultures jusque dans leurs déterminations les plus profondes ; elles proposent elles-mêmes de nouvelles valeurs ; elles apportent avec elles la possibilité objective d'un projet historique de la plus vaste envergure et de la plus haute qualité éthique. Comment les cultures peuvent-elles les accueillir sans se perdre, en répondant à la fois aux exigences de l'enracinement et de la finalisation et en accordant à la science et à la technologie toute leur part?

\section{LECOURT Dominique, Philosophie des sciences, PUF/Paris, 2015, 128 p.}

Les sentiments ambivalents qui entourent les progrès des sciences et la puissance croissante de leurs applications appellent une réflexion philosophique approfondie. Entre une confiance souvent aveugle et une inquiétude parfois excessive, comment trouver la voie de la raison ? Le XIX siècle a forgé le projet d'une "philosophie des sciences" pour faire face aux défis intellectuels et sociaux des sciences physico-chimiques. Cette discipline associe les compétences des scientifiques et des philosophes. L'ouvrage propose un tableau des doctrines qui se sont succédé et un état des débats actuels.

\section{TIERCELIN Claudine (dir.), La reconstruction de la raison: dialogues avec Jacques Bouveresse, Collège de France/Paris, 2014, OpenEditions Books, n.p. [en ligne]}

Autrefois supposée constituer ce qui unit les êtres humains, la raison n'a plus à présent de forme unitaire et dominante ; elle est au contraire fragmentée en une multitude de rationalités diverses, réelles ou supposées telles, qui coexistent de façon conflictuelle. L'époque a même été confrontée à un processus d'" irrationalisation de la science». Jusqu'à quel point et à quel prix la raison peut-elle espérer réussir à reconquérir une partie du rôle qu'elle a joué autrefois comme faculté d'unité et d'universalité ? L'entreprise présuppose une réponse suffisamment claire à la question : que peut-on conserver et que doit-on abandonner dans l'héritage des Lumières. L'ouvrage réunit les contributions au colloque organisé par la chaire Métaphysique et philosophie de la connaissance en mai 2013 au Collège de France.

[http://bit.ly/2BNJeyN]

\section{WILLIAMS Bernard, Vérité et véracité : essai de généalogie, Paris, Gallimard/} Paris, 2006, 384 p.

Sciences exactes et sciences humaines sont traversées par des conflits de vérité. La vérité existet-elle ou n'est-elle que relative, subjective ? L'auteur entend dénoncer des courants de pensée (relativisme, culturalisme, post-modernisme) qui n'accorderaient à la vérité qu'une valeur instrumentale. Pour présenter une défense de la vérité et des valeurs de vérité, il analyse comment se constituent ces dernières, en les exposant à travers divers épisodes historiques. L'humanité, dès sa première organisation sociale, et jusqu'aux démocraties contemporaines, a cherché à donner consistance à ces notions fondamentales que sont : la sincérité ou véracité, l'exactitude et l'authenticité.

\section{VÉRITÉ ET OPINION}

\section{IFOP, Enquête sur le complotisme, Fondation Jean Jaurés/Paris, 2017, 91 p. [en ligne]}

Selon cette enquête, le complotisme serait un phénomène social majeur dans la société française. Près d'un sondé sur dix est d'accord avec l'affirmation qu' « il est possible que la Terre soit plate et non pas ronde comme on nous le dit depuis l'école ». Comparativement à leurs aînés, les 
jeunes seraient nettement plus perméables aux "théories du complot». $31 \%$ des 18-24 ans adhérent à l'idée que «Dieu a créé l'homme et la Terre il y a moins de 10000 ans ». Ils sont quatre fois plus nombreux que l'ensemble des sondés à souscrire à une forme atténuée de négationnisme. [http://bit.ly/2Fakfox]

\section{KAVANAGH Jennifer, RICH Michael D, Truth decay: an initial exploration of the diminishing role of factory and educations and analysis in American public} life, RAND Corporation/Santa Monica, 2018, 301 p. [en ligne]

Ce rapport du think tank RAND corporation traite de la " dégradation de la vérité » aux ÉtatsUnis, qui serait le produit de quatre tendances : un désaccord croissant sur les faits et leur interprétation; un flou quant à la ligne de partage entre opinions et faits ; une augmentation du volume des opinions et de l'expérience personnelle sur les faits; et une baisse de la confiance dans les sources d'information. Il examine les causes de cette dégradation, dont la réduction du temps consacré à l'éducation aux médias et au développement de la pensée critique à l'école. [http://bit.ly/2okHTal]

\section{VÉRITÉ ET ÉDUCATION}

BORNE Dominique, Enseigner la vérité à l'école? Quels enjeux ? Armand Colin/ Paris, 2007, 160 p.

L'enseignement dispensé à l'école est-il vraiment un enseignement de vérité ? N'y a-t-il pas des sources de malentendu dans la différence entre la vérité établie et reconnue dans le contexte des diverses disciplines, les procédures d'élaboration des connaissances et l'acte pédagogique et la didactique ? Comment situer face aux élèves la recherche de la vérité de l'École républicaine ? Faut-il l'opposer à la liberté d'opinion et de croyance de chacun ? L'auteur examine quelques moments de l'histoire où ont été mis en scène des régimes de vérité (une vérité souvent présentée comme absolue), ainsi que l'histoire et la pensée du XXe siècle qui semblent relativiser toute connaissance.

\section{CARR David (éd.), Education, knowledge, and truth: beyond the postmodern impasse, Routledge/London, 1998, 267 p.}

La relation de la vérité et de la connaissance avec l'éducation est examinée en Angleterre, Écosse, Irlande, Pays-Bas, Canada, Afrique du Sud, Australie et aux États-Unis. Les auteurs mettent en cause "l'impasse post-moderne » selon laquelle il ne pourrait y avoir de discours commun consacrant des canons de rationalité, de connaissance et de vérité qui soient transculturels. Ils entendent réhabiliter une conception de la connaissance objective et de la vérité dans l'intérêt d'une théorisation cohérente de l'éducation et des curricula. Ils examinent l'influence du scepticisme et traitent de l'apprentissage, de la pensée critique, des valeurs et de l'évaluation dans le domaine de l'éducation.

FORQUIN Jean-Claude, "Savoirs scolaires, contraintes didactiques et enjeux sociaux », Sociologie des sociétés, vol. 23, $n^{\circ} 1$, 1991, p. 25-39

S'appuyant sur les apports de la sociologie du curriculum, l'auteur analyse certaines caractéristiques structurelles des savoirs scolaires dans les sociétés contemporaines. Il examine les sélections opérées au sein de la culture pour constituer les programmes scolaires, les effets de la contrainte didactique et les traits de la structuration des savoirs, et les phénomènes de légitimation sociale. Une telle approche analytique et critique du curriculum implique-t-elle nécessairement une conception relativiste de la connaissance et de la culture ? Est-elle compatible avec l'exigence pédagogique d'une justification "normative » des contenus d'enseignement? 
MARIN Brigitte, SAVATOVSKY Dan (dir.), Épistémologie, savoirs et champs disciplinaires : questions d'apprentissage, CRDP de l'académie de Créteil/Créteil, 2013, $128 p$.

L'introduction d'une réflexion épistémologique met au jour des questions transversales aux disciplines : le problème des taxinomies du mot discipline, ses enjeux sociaux ainsi que l'introduction de la réflexivité. Ce qui conduit à s'interroger sur le passage du savoir constitué par une communauté scientifique au savoir enseigné, détermine des contenus disciplinaires et influence le rapport aux savoirs de l'enseignant. Les contributions sont issues de la conférence de consensus « Épistémologie, savoirs et champs disciplinaires » organisée en 2011 par l'académie de Créteil.

\section{MATTHEWS Michael R., Science teaching, the contribution of history and philo-} sophy of science, Routledge/New York, 2015, 454 p.

L'auteur examine dans une perspective historique comment l'histoire et la philosophie des sciences contribuent à résoudre des problèmes d'ordre théorique, curriculaire et pédagogique dans l'enseignement des sciences. Il examine les fondements de la connaissance scientifique et ses liens avec la philosophie, la métaphysique, les mathématiques, ainsi que ses dimensions sociales et montre pourquoi il est essentiel que les professeurs de sciences connaissent l'histoire et la philosophie.

\section{Diversité, ÉCOLE et RELIgIONS}

AKKARI Abdeljalil (coord.), "L'école et la diversité de cultures », Revue internationale d'éducation Sèvres, septembre 2013, n 63, p. 33-162 [en ligne]

Depuis plusieurs décennies, la prise en compte de la diversité des cultures à l'école gagne en audience sinon en légitimité à différents niveaux des systèmes éducatifs. Des doutes et des interrogations subsistent pourtant quant à la pertinence, à l'impact et à la mise en œuvre de cette prise en compte dans le quotidien scolaire. Quels sont, selon les contextes, les choix et les approches privilégiés ? Les articles illustrent l'actualité du débat dans plusieurs pays : Algérie, Brésil, France, Malaisie, Norvège, Portugal, Canada (Québec), Suisse, ainsi que dans les instances internationales. [http://bit.ly/2BhTSNQ]

BISSON-VAIVRE Claude, BOISSINOT Alain (coord.), "Laïcité, école et religions ", Administration et éducation, $n^{\circ} 148,2015$, p. 3-195

L'institution scolaire est-elle encore à la hauteur de sa mission fondatrice, former le citoyen? Comment le débat sur les valeurs de la République pénètre-t-il dans les classes ? Comment ces valeurs et leurs approches sont-elles perçues par les élèves ? À quelles conditions les enseignants peuvent-ils s'engager dans leur défense ? Les articles interrogent la référence à la laïcité et aux valeurs républicaines, et réfléchissent à la place du religieux dans une école laïque. La situation française est éclairée par celles de pays de culture proche ou plus éloignée (Allemagne, Belgique francophone, Japon).

BOURGEAULT Guy, GAGNON France, "L'espace de la diversité culturelle et religieuse à l'école dans une démocratie de tradition libérale ", Revue Européenne des Migrations Internationales, 11-3, 1995, p. 79-103

L'article explore l'enjeu complexe que posent aux institutions publiques les demandes de plus en plus fréquentes de prise en compte de la diversité culturelle et religieuse. Il analyse les fondements et les limites du rôle de l'État face à celle-ci. Rejetant la position libérale traditionnelle de neutralité axiologique comme la position communautarienne, les auteurs mettent en avant une approche d'accueil critique des différences balisée par le principe du respect de l'indépendance morale de l'individu et des valeurs démocratiques fondamentales. Ils discutent du rôle de l'école dans la formation civique et dans la transmission d'un curriculum où normes communes et diversité auraient également droit de cité. 
FAAS Daniel, "The Nation, Europe and migration: a comparison of geography, history, and citizenship education curricula in Greece, Germany, and England ", Journal of curriculum studies, vol. $43 n^{\circ}$ 4, août 2011, p. 471-492

Les programmes scolaires nationaux sont contestés et transformés par l'impact de la migration et de l'intégration européenne. L'auteur examine comment la diversité culturelle et l'Europe sont liées dans les programmes scolaires d'histoire, de géographie et d'éducation à la citoyenneté en Grèce, en Allemagne et en Angleterre. L'analyse transnationale montre que les relations entre les valeurs européennes et multiculturelles sont agencées différemment selon les pays et les disciplines.

\title{
LANTHEAUME Françoise (dir.), "Les religions à l'école ", Histoire, monde et cultures religieuses, $n^{\circ} 32,2014,184 p$.
}

Privilégiant une approche pluridisciplinaire et internationale, le dossier étudie les interprétations des principes structurant le rapport entre école et religions et l'action des professionnels de l'éducation. Il aborde les points litigieux dans le quotidien de l'école, ainsi que les approches et pratiques des différents acteurs qui varient selon les traditions nationales. Si, en France, la question est rapportée à l'interprétation de la laïcité, elle est dominée au Québec par la notion $\mathrm{d}^{\prime}$ ' accommodements raisonnables ». Partout le débat public est vif et porte sur les conditions du « vivre ensemble », les finalités de l'école, son organisation et les contenus d'enseignement.

\begin{abstract}
MABILON-BONFILS Béatrice, ZOIA Geneviève (coord.), "École, citoyenneté, ethnicité », Recherches en éducation, $n^{\circ} 26,2016$, p. 2-111 [en ligne]

Quel sens revêt la notion d'identité selon le contexte culturel ? Comment permet-elle d'appréhender la fabrique du sentiment national ? En quoi l'institution scolaire est-elle encore partie prenante d'un processus de construction identitaire et dans quelle mesure ce processus est-il intégrateur? Les articles interrogent la question de la formation et de la cohabitation des identités dans les sociétés contemporaines, sous l'angle de différentes disciplines en France, Belgique et à Maurice. [http://bit.ly/2EPLgjN]
\end{abstract}

\section{CONTROVERSES À L’ÉCOLE}

CROCHÉ Sarah (dir.), "Concurrence des discours de vérité à l'école », Éducation et société, $n^{\circ} 33,2015$, p. 5-136

Les articles s'intéressent à la façon dont les systèmes éducatifs, les programmes, les manuels, les enseignants et les élèves tentent de concilier le discours de la science dite occidentale avec les discours issus de la religion, de l'opinion et de la tradition. Ils traitent de la question des identités, des croyances, du pouvoir et des résistances à l'école moderne dans différents pays (Belgique, Djibouti, France, Grèce, Sénégal, Tunisie, Rwanda), ainsi que dans une enquête internationale L'idée de concurrence des discours de vérité renvoie aux écrits de Bruno Latour pour qui notre société moderne n’a jamais fonctionné conformément au grand partage qui fonde son système de représentation du monde.

\section{GRANDJEAN Geoffrey, PIET Grégory (dir.), Polémiques à l'école : perspectives internationales sur le lien social, Armand Colin/Paris, 2012, 200 p.}

Lieu d'échanges et de formation des citoyens, l'école est marquée par la double rencontre entre socialisation et polémiques multiples. C'est aussi une arène publique au sein de laquelle le phénomène de politisation est toujours parvenu à se manifester, que ce soit dans les livres d'histoire ou dans le choix des événements à étudier ou non. En prenant appui sur des études de cas français, belges, canadiens, américains, espagnols et libanais, l'ouvrage analyse les formes de lien social et les tensions du vivre ensemble à l'école dans le contexte de la globalisation. Les auteurs se concentrent en particulier sur la place de la religion, son enseignement et les concurrences mémorielles. 


\section{Sciences et religion}

AROUA Sä̈da, COQUIDÉ Maryline, ABBES Salem, "Controverses dans l'enseignement de l'évolution : questions de recherche sur les stratégies d'intervention en classe et dans la formation ", RDST : Revue des sciences et des technologies, $n^{\circ} 5$, 2012, p. 47-76 [en ligne]

Une campagne anti-évolutionniste s'est amplifiée depuis trois décennies dans certains pays où la théorie de l'évolution a de moins en moins de place dans les programmes scolaires et où sa compréhension diminue chez les élèves. L'article passe en revue la littérature internationale sur l'enseignement de l'évolution et présente des recherches pédagogiques et didactiques prenant en compte les tensions possibles dans trois pays : les Philippines, la Tunisie, les États-Unis. Les stratégies décrites incitent à traiter d'éventuelles tensions afin de cultiver un esprit critique afin de renforcer le statut scientifique de l'enseignement de l'évolution. [http://bit.ly/2Eqsu2e]

\section{COQUIDÉ Maryline, TIRARD Stéphane (coord.), L'évolution du vivant : un ensei-} gnement à risque ?, Vuibert/Paris, 2009, 226 p.

L'enseignement de la théorie de l'évolution devient parfois une "question sensible ", quand des groupes religieux la contestent. L'offensive des mouvements néo-créationnistes à l'école appelle un travail d'épistémologie auquel les enseignants sont peu préparés. Il s'agit, selon les auteurs, de mettre en place une formation à l'esprit critique où le respect des cultures et des opinions ne conduira ni à esquiver le débat, ni à renoncer à réfléchir avec rigueur. L'ouvrage examine la réalité de l'enseignement de l'évolution aujourd'hui, la nature des obstacles didactiques ou socioculturels; il rappelle l'apport cladistique afin de soutenir les professeurs ou d'aider les étudiants à mieux assimiler la biologie contemporaine.

FORTIN Corinne, "L'enseignement de l'évolution face aux croyances religieuses : quelles perspectives curriculaires possibles? ", HMC: Histoire, monde et cultures religieuses, $n^{\circ} 32,2014$, p. 67-78

En France, les instructions officielles concernant l'enseignement de la biologie de l'évolution préconisent d'ignorer les considérations religieuses que pourraient exprimer les élèves. Cet article s'appuie sur l'histoire curriculaire de l'enseignement de l'évolution, du XIX ${ }^{\mathrm{e}}$ au XX $\mathrm{XX}^{\mathrm{e}}$ siècle pour explorer de nouvelles pistes qui prendraient en compte le questionnement religieux des élèves sans renoncer à l'ambition de l'École laïque de s'adresser à tous dans le respect des convictions ou des croyances de chacun.

Kebaïli Sonia, AZZOUNA Atf, "Hétérotopie et problématisation non formelle : débat sur l'évolution biologique à la Cité des sciences de Tunis ", Recherches en didactique des sciences et des technologies, $n^{\circ}$ 13, 2016, p. 221-248

Dans le contexte concordiste de la Tunisie, marqué par la montée de l'intégrisme religieux, aborder l'évolution biologique dans un cadre scolaire ou médiatique devient de plus en plus difficile. Ce constat conduit à ne plus limiter le traitement didactique de la théorie de l'évolution à sa dimension cognitive, mais à le considérer comme une question assujettie à divers pouvoirs. L'article examine un dispositif de rencontre d'une classe et d'un scientifique pour un débat à la Cité des sciences de Tunis qui a permis de progresser dans la manière de problématiser l'histoire du vivant chez des lycéens.

MACHADO SILVA Heslley, FLEURY MORTIMER Eduardo, EVILIN GIBRAL Daiana, et al., "Perspective of science and religion in high school biology teachers in Argentina, Brazil and Uruguay: a comparative study », Procedia - Social and Behavioral Sciences, $n^{\circ}$ 197, 2015, p. 780-787 [en ligne]

L'article analyse quelques résultats d'une étude comparative réalisée en Amérique latine dans trois pays présentant des niveaux différents de sécularisation (Argentine, Brésil, Uruguay) sur 
les conceptions des enseignants de biologie du rapport entre la religion et la science et les origines de la vie. L'enquête utilisait le questionnaire du projet européen BIOHEAD-CITIZEN. Une grande majorité d'enseignants argentins et uruguayens sont évolutionnistes, même lorsqu'ils sont croyants. La plupart des enseignants brésiliens adoptent une position religieuse, qu'ils soient créationnistes ou créationnistes évolutionnistes. [http://bit.ly/2CO2vwY]

SOLOMON TSEHAYE Rachel, "Les régimes de cohabitation des discours de vérité religieux et scientifiques dans les écoles djiboutiennes ", Carrefours de l'éducation, $n^{\circ} 44,2017$, p. 84-99

Cet article vise à éclairer les conflits de normes imputables aux spécificités contextuelles à Djibouti, où cohabitent dogme religieux dans la société et, à l'école, laïcité et science. En déconstruisant la subalternité des connaissances, l'auteure interroge les formes d'interprétation, d'adhésion, de résistances au discours de vérité scientifique. L'analyse des données met à jour trois différents régimes de recherche d'unité de sens, à travers les stratégies de rejet ou d'accommodements opérées par les individus.

\section{WOLFS José Luis, Sciences, religions et identités culturelles : quels enjeux pour} l'éducation? De Boeck/Bruxelles, 2013, 343 p.

On observe chez les élèves rejet, confusion ou amalgame entre le registre des sciences et celui des croyances religieuses. Ces phénomènes ne se limitent pas aux États-Unis, ou à certains pays du monde musulman ou d'Afrique, et soulèvent de nombreuses questions, sur le plan épistémologique, et sur le plan sociétal (comment garantir la liberté de conscience et éviter l'immixtion des croyances dans le champ des savoirs scientifiques ?). L'ouvrage présente un cadre théorique, des exemples de situations problématiques et des recherches réalisées dans différentes régions du monde, ainsi qu'une enquête menée en Belgique francophone et des pistes de réflexion sur le plan pédagogique et didactique.

\section{Quelques exemples en histoire}

FALAIZE Benoit, «L'enseignement des sujets controversés dans l'école française : les nouveaux fondements de l'histoire scolaire en France? ", Revista Tempo $\boldsymbol{e}$ Argumento, vol. 6, $n^{\circ} 11,2014$, p. 193-223 [en ligne]

Shoah, colonisation, esclavage, questions religieuses, certains sujets qui touchent à l'identité ou à l'intime, suscitent parfois le débat en classe. L'auteur examine la problématique de l'enseignement des questions sensibles de l'histoire qui émerge depuis une vingtaine d'années en France. Il analyse le tournant mémoriel des années 1980 observable dans les prescriptions scolaires, pour examiner la place qu'ont en classe les traumatismes de l'histoire nationale, la manière dont l'histoire est convoquée, et la réaction des élèves en face de tels sujets sensibles. [http://bit.ly/2C4foCF]

KELLO Katrin, WAGNER Wolgang, "Intrinsic and extrinsic patriotism in school: teaching history after Estonia's critical juncture ", International Journal of Intercultural Relations, vol. 43, 2014, p. 48-59

En Estonie, où vit, outre la majorité ethnique estonienne, une importante minorité de russophones, les deux communautés abordent le passé estonien selon des perspectives fort différentes. L'étude porte sur la façon dont les professeurs d'histoire des deux groupes traitent les attentes de la société et de l'État relativement au patriotisme à inculquer à leurs élèves. Alors que les enseignants estoniens poursuivent ce but d'une manière largement implicite, les enseignants russes perçoivent beaucoup plus explicitement les attentes de l'État et s'efforcent de bâtir la loyauté de leurs étudiants à l’Estonie. 
ZADORA Anne, "L'enseignement de questions vives en Biélorussie : enjeux et spécificités", Questions vives : recherches en éducation, $n^{\circ} 26,2016$, n.p.

L'usage de l'enseignement de l'histoire dans l'objectif de légitimer un système politique n'est pas une spécificité de l'espace post-soviétique. En Biélorussie, à l'époque soviétique, il visait une mémorisation mécanique des vérités dogmatiques. Mobilisée pour légitimer le projet politique sous la perestroïka, l'histoire scolaire est devenue une discipline indépendante et pour la première fois l'objectif de former des citoyens et d'éveiller l'esprit critique a été évoqué. Les manuels scolaires ont été réécrits après la perestroïka pour mieux répondre aux finalités politiques imposées au système éducatif, repoussant la pluralité de l'enseignement historique à une interprétation historique présentée comme une vérité ultime.

\title{
ENSEIGNER (OU PAS) LES CONTROVERSES
}

\begin{abstract}
ALBE Virginie, Enseigner des controverses, Presses universitaires de Rennes/ Rennes, 2009, 223 p.

L'ouvrage propose une synthèse internationale sur la question des controverses et des questions vives - vives au sein de la communauté scientifique, et/ou vives dans la société. On assisterait à la fin du compromis historique entre sciences et société au profit d'un nouvel équilibre fondé sur la privatisation des savoirs et de l'éducation, ce qui change aussi bien la nature des sciences que les discours à leur sujet. Ces évolutions justifient, pour l'auteure, l'introduction des questions socialement vives à l'école : les technosciences étant constitutives de la société, « le travail des questions socialement vives peut contribuer à un enrichissement de la vie sociale et au développement d'une citoyenneté démocratique».
\end{abstract}

CAVET Agnès, "L'enseignement des “questions vives" : lien vivant, lien vital, entre école et société ? », Lettre d'information de la VST, $n^{\circ}$ 27, mai 2007, 18 p. [en ligne] Jusqu'à encore récemment, la plupart des disciplines scolaires étaient données à enseigner sur un modèle positiviste, transmettant des "vérités objectives» sur l'organisation du monde naturel, de la société et de ses produits. S'appuyant sur une revue de la littérature française et internationale, le dossier vise à comprendre les particularités épistémologiques et didactiques des questions vives. L'auteure en propose un tour d'horizon à travers différents champs disciplinaires afin de prendre la mesure des pratiques d'enseignement. La place croissante du débat comme moyen d'apprentissage est aussi explorée. [http://bit.ly/2Hecn72]

\section{CCEA: Council for the curriculum, examinations and assessment, Teaching contro-} versial issues at key stage 3, CCEA /Belfast, 2015, 66 p. [en ligne]

L'Irlande du Nord demeure une société divisée ; elle est aussi plus diversifiée et multiculturelle. Nombre de questions liées au passé récent du pays divisent l'opinion publique et se répercutent à l'école. Aborder l'enseignement de questions controversées peut être difficile. Assorties d'exemples dans les différentes disciplines - notamment l'histoire et l'éducation à la citoyenneté mondiale, ces lignes directrices visent à soutenir les enseignants et promouvoir une approche globale de l'enseignement des questions controversées dans les établissements scolaires. Elles recommandent de favoriser la discussion, la gestion des émotions, de développer la pensée critique des élèves, de promouvoir l'empathie, et d'associer les familles. [http://hit.ly/1W3YMCg]

KERR David, HUDDLESTON Ted (éd.), Living with controversy: teaching controversial issues through education for democratic citizenship and human rights (EDC/ HRE): training pack for teachers, Council of Europe/Strasbourg, 2015, 72 p. [en ligne] Apprendre à nouer le dialogue avec les personnes qui cultivent des valeurs différentes et à les respecter est une démarche essentielle au renforcement de la démocratie. Fondé sur une analyse 
de publications internationales, ce guide vise à soutenir et à promouvoir l'enseignement des sujets controversés dans tous les contextes éducatifs et dans toutes les matières - de l'éducation à la citoyenneté et sciences sociales aux langues et aux sciences. Les enseignants sont invités à se pencher sur l'influence qu'exercent leurs convictions et valeurs. Une approche ouverte et collaborative de l'enseignement est prônée. L'accent est mis sur le développement l'esprit critique. [http://bit.ly/2DPXW6D]

\section{LEGARDEZ Alain, SIMMONEAUX Laurence, (dir.), L'école à l'épreuve de l'actua-} lité : enseigner les questions vives, ESF/Paris, 2006, 246 p.

Peut-on parler du conflit israélo-palestinien dans les manuels scolaires ? Comment aborder la question du genre et de l'égalité des sexes avec les élèves? Comment garder du recul et une certaine neutralité ? L'ouvrage se penche sur les questions socialement vives (QSV) - vives dans la société, dans les savoirs de référence, et dans les savoirs scolaires. Les contributions traitent des disciplines scientifiques expérimentales et des sciences humaines et sociales. Elles examinent les risques didactiques, la question de la légitimité des savoirs et montrent que les QSV remettent en question les frontières disciplinaires.

PHILPOTT Sarah, CLABOUGH, MCCONKEY Lance, et al., "Controversial issues: to teach or not to teach? That is the question! ", The Georgia Social Studies Journal, 2011, vol. 1, $n^{\circ} 1$, p. 32-44

Quelles sont les perceptions, les croyances et les pratiques des enseignants liées à l'enseignement des sujets controversés aux États-Unis ? Les enseignants les abordent-ils ? À quel moment? Comment réagissent les élèves? Les sujets controversés ont souvent trait à la religion, à l'évolution biologique, à l'histoire, à la politique. S'appuyant sur une revue de la littérature et une série d'entretiens menés auprès d'enseignants du secondaire au Tennessee, l'article souligne l'inconfort et le manque de préparation des enseignants, ainsi que le besoin des élèves de discuter des sujets sensibles.

WOOLLEY Catherine Mary, "The attitudes and perceptions of beginning teachers in relation to teaching controversial and sensitive issues in the history classroom ", Revista Electrónica Interuniversitaria de Formación del Profesorado, vol. $20, n^{\circ} 2,2017,16 p$.

En Grande-Bretagne, un rapport officiel sur l'enseignement des questions sensibles en classe d'histoire avait montré en 2007 que des enseignants évitaient certains sujets. L'article analyse les résultats d'une nouvelle étude sur les perceptions des enseignants débutants : ils semblent déterminés à partager un large éventail de points de vue avec leurs élèves et apparaissent confiants. Presque tous les enseignants pensent qu'il est justifié d'aborder des sujets entrant en conflit avec les valeurs familiales ou culturelles. Certains demeurent circonspects quant à la manière d'enseigner l'histoire des migrations.

\section{L'enseignement des controverses socio-scientifiques}

ALBE Virginie, GOMBERT Marie-José, "Intégration scolaire d'une controverse socioscientifique contemporaine : savoirs et pratiques d'élèves pour appréhender les savoirs et pratiques de scientifiques ", Recherches en didactique des sciences et des technologies, $n^{\circ} 2,2010, p .103-126$ [en ligne]

Des recherches sur l'argumentation entre élèves sur des controverses socioscientifiques indiquent que des connaissances scientifiques sont rarement mobilisées. Des connaissances épistémologiques renouvelées permettraient aux élèves de ne pas interpréter les désaccords en termes 
d'intérêts. L'article présente une recherche sur l'intégration scolaire d'une controverse socioscientifique contemporaine, le réchauffement climatique. La compréhension de la science comme une activité sociale ferait comprendre aux élèves comment les savoirs scientifiques sont collectivement établis. [http://bit.ly/2CYhlAT]

REIS Pedro, "Factors of success of SSI Regarding classroom discussions: a crosscase study ", Les Dossiers de sciences de l'éducation, $n^{\circ}$ 29, 2013, p. 67-80 [en ligne]

La discussion des questions socio-scientifiques dans l'enseignement des sciences est préconisée, notamment afin de développer des compétences essentielles à une citoyenneté active. Cependant, elle est absente de nombreux cours, même lorsque les questions socio-scientifiques figurent dans les programmes et que les enseignants valorisent leur discussion. L'article analyse une série d'études centrées sur les pratiques des enseignants portugais de sciences naturelles. Il vise à identifier les facteurs qui influencent positivement la discussion de questions socioscientifiques en classe. [http://bit.ly/2CYhlAT]

\section{VERS LA FORMATION DU CITOYEN?}

\section{Études transnationales}

\section{BANKS James (dir.), A citizenship education and global migration: implications} for theory, American Educational Research Association/Washington, 2017, 572 p.

L'ouvrage examine les fondements théoriques, les travaux issus de la recherche et les approches pratiques mobilisables dans les cours d'éducation civique pour aider les élèves des groupes marginalisés et issus de minorités à acquérir un sentiment d'intégration. Seize études de cas (Afrique du Sud, Allemagne, Angleterre, Brésil, Canada, Chine, Corée du Sud, États-Unis, France, Mexique, Norvège, Singapour, et pays du Moyen-Orient) analysent, dans le contexte de leur pays, les difficultés des enseignants, et présentent des stratégies pédagogiques innovantes favorisant l'inclusion, la participation civique et politique, et le renforcement des identités culturelles, nationales et mondiales.

GAUSSE Marie, "Développer l'esprit critique par l'argumentation : de l'élève au citoyen ", Dossier de veille de l'IFÉ, $n^{\circ}$ 108, février 2016, 24 p. [en ligne]

Depuis quelques années l'argumentation reprend place dans les programmes scolaires de nombreux pays, tant comme objet d'apprentissage que comme élément d'une démarche critique. En Angleterre et en France par exemple, savoir argumenter semble moins primordial que les contenus disciplinaires, alors qu'aux États-Unis et en Écosse, la rhétorique et l'argumentation sont considérées comme des habiletés à enseigner. Le dossier s'intéresse au développement de la pensée critique, à l'impact des procédés argumentatifs sur la forme scolaire et sur la formation des élèves comme futurs citoyens. [http://bit.ly/2Euhlbi]

\section{MISCO Thomas, DE GROOF Jan (éd.), Cross-cultural case studies of teaching} controversial issues: pathways and challenges to democratic citizenship education, Wolf Legal Publishers/Oisterwijk, 2014, 274 p.

Les contributions explorent l'enseignement des questions controversées dans différents contextes nationaux (États-Unis, Australie, Chine, Afrique du Sud, Ghana, Kenya, Macédoine, Irlande du Nord, Philippines, Singapour, Corée du Sud et Turquie). Les questions qu'elles soulèvent sont au cœur des programmes d'éducation à la citoyenneté et ont une influence profonde sur la viabilité des sociétés démocratiques, tant il est essentiel que les élèves débattent du bien commun 
et prennent position à partir de multiples sources et points de vue. L'ouvrage propose des analyses théoriques et pratiques transférables dans la perspective d'un renforcement de l'éducation à la citoyenneté.

MOURITSEN Per, JEAGER Astrid, Designing civic education for diverses societies: models, tradeoffs, and outcomes, Migration Policy Institute Europe/Bruxelles, 2018, 28 p. [en ligne]

L'intérêt renouvelé pour l'éducation civique reflète la montée de nouveaux défis : désengagement politique des jeunes, intégration des réfugiés et des enfants issus de l'immigration, tensions ethniques. Ces dernières années, sa place dans les programmes d'enseignement a été renforcée pour combler le déficit civique et renforcer le sens des valeurs communes. En examinant la diversité des modèles nationaux (Danemark, France, Allemagne, Suède et RoyaumeUni), le rapport analyse ces initiatives et leurs effets. Il montre comment les modèles traduisent des conceptions différentes de la notion de «bon » citoyen. Il étudie les compromis, les tensions entre buts et méthodes. [http://bit.ly/2CDCakY]

\section{Études de cas}

AUDIGIER François, "Débat et formation politique : interrogation sur une nouvelle norme pédagogique : autour de l'exemple de l'éducation en vue du développement durable » in ÉTHIER Marc-André, MOTTET Éric (dir.)., Didactiques de l'histoire de la géographie et de l'éducation à la citoyenneté : recherches et pratiques, De Boeck/Bruxelles, 2016, p. 129-137

S'appuyant sur une recherche menée en Suisse romande, l'auteur interroge la pertinence du débat en classe - dispositif promu depuis plusieurs décennies - dans la construction de la conscience citoyenne chez l'élève - à travers les questions socialement vives, et plus particulièrement le concept de développement durable. Il souligne la transformation des conceptions des débats scolaires, de leurs formes et de leurs objets, qui renouvellerait la formation du citoyen de l'élève.

BOUCHARD Nancy, DANIEL Marie-France, DESRUISSEAUX Jean-Claude, "L'éducation éthique dans les orientations du programme québécois Éthique et culture religieuse : une éducation au vivre-ensemble?", Éducation et francophonie, vol. XLV, $n^{\circ} 1,2017$, p. 60-88

Le défi de la coexistence est l'objet de nombreux débats sur le vivre-ensemble dans la diversité. Les auteurs analysent le programme québécois Éthique et culture religieuse et montrent qu'une éducation au vivre-ensemble est présente sous forme de pratique de délibération dans le dialogue et sous forme d'acquisition de connaissances sur les cultures religieuses. Ils soulignent que le vivre-ensemble s'apprend notamment en sollicitant la dimension cognitive et rationnelle de l'élève en tant que citoyen.

BICKMORE Kathy, PARKER Christina, "Constructive conflict talk in classrooms: divergent approaches to addressing divergent perspectives ", Theory \& Research in Social Education, vol. 42, $n^{\circ} 3,2014$, p. 291-335

Au Canada, le dialogue sur les conflits sociaux et politiques est un élément clé de l'éducation à la citoyenneté démocratique, souvent préconisée, mais rarement pleinement mise en œuvre, en particulier dans les classes ethniquement et économiquement hétérogènes. L'article analyse différentes approches mises en œuvre par des enseignants. Les leçons introduisent des compétences de communication de conflit incarnant des points de vue contradictoires et concernent un large éventail d'élèves. Cependant, reconnaître des points de vue divergents et des conflits sociaux ne débouche pas toujours sur un dialogue démocratique durable. 
DAVIES Lynn, «Teaching about conflict through citizenship education », International journal of citizenship and teacher education, vol. 1, $n^{\circ} 2,2005, p .17-34$ S'appuyant sur les résultats d'un projet du Centre d'éducation et de recherche internationales de Birmingham sur les besoins de enseignants et apprenants pour l'éducation à la citoyenneté mondiale, l'auteure présente dix façons d'aborder ou d'éviter en cours la guerre et les conflits, depuis les postures les plus susceptibles d'exacerber les tensions, en passant par les positions neutres, jusqu'à celles qui favorisent l'apaisement. Les moyens de faire progresser l'éducation à la paix dans l'agenda des écoles sont explorés.

HANNA Helen, "Dealing with difference in the divided educational context: balancing freedom of expression and non-discrimination in Northern Ireland and Israel ", Compare: A Journal of Comparative and International Education, vol. $47 n^{\circ}$ 1, 2017, p. 17-31

Une éducation à la citoyenneté efficace dans une société multiculturelle suppose une confrontation avec une variété de points de vue. Mais la capacité et la volonté de traiter les différences liées à des questions d'identité nationale, de récit et de conflit, peuvent varier considérablement. L'article étudie la gestion des différences dans l'éducation à la citoyenneté en Irlande du Nord et en Israël. En se fondant sur le droit international en éducation, il examine comment la liberté d'expression et la non-discrimination sont interprétées et équilibrées différemment quand, dans une classe d'une société divisée, sont abordées des questions controversées. 